\title{
Aged-Related Neural Changes During Memory Conjunction Errors
}

\section{Citation}

Giovanello, Kelly S., Elizabeth A. Kensinger, Alana T. Wong, and Daniel L. Schacter. Forthcoming. Aged-related neural changes during memory conjunction errors. Journal of Cognitive Neuroscience 22.

\section{Published Version}

doi:10.1162/jocn.2009.21274

\section{Permanent link}

http://nrs.harvard.edu/urn-3:HUL.InstRepos:3627273

\section{Terms of Use}

This article was downloaded from Harvard University's DASH repository, and is made available under the terms and conditions applicable to Other Posted Material, as set forth at http:// nrs.harvard.edu/urn-3:HUL.InstRepos:dash.current.terms-of-use\#LAA

\section{Share Your Story}

The Harvard community has made this article openly available.

Please share how this access benefits you. Submit a story.

\section{Accessibility}




\title{
Aged-related Neural Changes during Memory Conjunction Errors
}

\author{
Kelly S. Giovanello ${ }^{1}$, Elizabeth A. Kensinger ${ }^{2}$, \\ Alana T. Wong ${ }^{3}$, and Daniel L. Schacter ${ }^{4}$
}

\begin{abstract}
Human behavioral studies demonstrate that healthy aging is often accompanied by increases in memory distortions or errors. Here we used event-related functional MRI to examine the neural basis of age-related memory distortions. We used the memory conjunction error paradigm, a laboratory procedure known to elicit high levels of memory errors. For older adults, right parahippocampal gyrus showed significantly greater activity during false than during accurate retrieval. We observed no regions in which activity was greater during false than during
\end{abstract}

\section{INTRODUCTION}

Memory is not an exact replay of the past. Instead, memory depends on constructive processes that are occasionally susceptible to illusions, errors, or distortions (Schacter \& Slotnick, 2004; Schacter, 2001; Roediger \& McDermott, 2000). Importantly, a growing number of studies have documented that older adults can be more susceptible to various kinds of memory distortions than are younger adults (Jacoby \& Rhodes, 2006; Dodson, Koutstaal, \& Schacter, 2001). For example, studies of false recognition, where people incorrectly claim that they have recently encountered a novel item or event and express high confidence in these false claims (Underwood, 1965), have revealed striking age-related increases in memory errors (e.g., Dodson \& Schacter, 2002; Tun et al., 1998; Jennings \& Jaocby, 1997; Koutstaal \& Schacter, 1997; Norman \& Schacter, 1997).

A type of false recognition procedure known as the memory conjunction error paradigm, developed initially by Underwood and Zimmerman (1973), has also revealed clear evidence for aging effects. Memory conjunction errors represent a form of memory illusion in which participants falsely claim to recognize an item because all of its constituent components were contained across several previously presented items. For example, following presentation of study words such as "blackmail," "jailbird," and "shoestring," participants may subsequently claim to recognize conjunction lures (e.g., "blackbird"-in which both parts are studied, yet recombined) and feature lures (e.g., "draw-

\footnotetext{
${ }^{1}$ The University of North Carolina at Chapel Hill, ${ }^{2}$ Boston College,

${ }^{3}$ The University of California at Berkeley, ${ }^{4}$ Harvard University
}

accurate retrieval for young adults. Young adults, however, showed significantly greater activity than old adults during accurate retrieval in right hippocampus. By contrast, older adults demonstrated greater activity than young adults during accurate retrieval in right inferior and middle prefrontal cortex. These data are consistent with the notion that age-related memory conjunction errors arise from dysfunction of hippocampal system mechanisms, rather than impairments in frontally-mediated monitoring processes.

string" - in which one part is studied and one part is novel). In this paradigm, memory failures lead participants to endorse as "old" conjunction and feature lures because such stimuli consist of studied elements-although not the specific combination of elements seen at study. Conjunction errors have been observed across of a wide set of stimulus materials including compound and noncompound words, nonsense words, word pairs, sentences, pictures, and faces (Jones \& Jacoby, 2005; Rubin, Van Patten, Glisky, \& Newberg, 1999; Jha, Kroll, Baynes, \& Gazzaniga, 1997; Kroll, Knight, Metcalfe, Wolf, \& Tulving, 1996; Reinitz, Verfaellie, \& Milberg, 1996; Dorfman, 1994; Reinitz, Lammers, \& Cochran, 1992; Underwood, Kapelak, \& Malmi, 1976) and in a variety of participant groups including healthy young adults and older adults (Jones \& Jacoby, 2005; Rubin et al., 1999; Kroll et al., 1996) as well as several neuropsychological patient populations (Rubin et al., 1999; Jha et al., 1997; Kroll et al., 1996).

To date, the mnemonic processes underlying agerelated conjunction errors remain unclear. One proposal is that these errors reflect a binding failure during encoding, such that elements of an initial learning event are inappropriately recombined into episodes that did not actually take place (Kroll et al., 1996). Binding failures may arise from weak and inefficient binding of stimulus elements, perhaps reflecting a failure to encode relational information. This proposal was suggested by Kroll et al. (1996), who conducted two experiments with undergraduate students, older adults, and patients with severe memory impairment (following damage to the hippocampal system). Results from both experiments revealed that older 
adults, and to an even greater extent memory impaired patients, showed a lower rate of correctly endorsing stimuli presented at study (i.e., hit rates) as well as higher rates of memory conjunctions errors (i.e., conjunction lures) compared with young adults. The results were taken as evidence that older adults and hippocampal patients may encode and store the components of stimuli but not the relationship among these components (i.e., a binding deficit at encoding).

Such an account proposes two types of representations: features and conjunctions (Jones, Jacoby, \& Gellis, 2001). Whereas features are the basic components or representations of stimuli, configural representations arise when two or more features are bound together. For example, the word "shoestring" consists of two separate features, "shoe" and "string," as well as the configural presentation of "shoestring." According to this representational account, age-related conjunction errors arise either from feature representations in the absence of configural representations or inaccurate configuration representations formed at encoding (Kroll et al., 1996).

A contrasting proposal is that age-related conjunction errors arise from faulty monitoring processes at retrieval. Proponents of this processing account suggest that individuals mistake the ease in processing of studied elements (i.e., processing fluency) with familiarity of the whole word and as a consequence endorse as "old" conjunction and feature lures (Rubin et al., 1999). Because conjunction and feature lures engender more familiarity than new words, such lures are identified as "old" more often than baseline. Rubin et al. (1999) examined memory conjunction errors in young and older adults who were characterized according to their neuropsychological status. The results indicated that neuropsychological tasks sensitive to executive functions (i.e., frontal lobe function) predicted conjunction error rates but not correct responses. An additional electrophysiological study of younger adults using event-related potentials provided evidence that memory conjunction errors are retrieved differently than correct responses. Based on these collective findings, the authors suggested that lures to conjunction lures are products of faulty, frontally mediated monitoring processes during retrieval.

More recently, Jones and Jacoby (2005) extended the processing view by suggesting that in some instances, individuals may avoid a conjunction error at test by remembering that a different word was presented at study. They cast this proposal within the context of a dual process theory of recognition, which postulates two bases for recognition judgments: recollection of the study episode and familiarity with the test probe. Although familiarity may typically lead to conjunction errors, if individuals can recollect the studied words, that recollection should counteract the effects of fluency.

Jones and Jacoby (2005) explored the hypothesis that older adults' inability to recollect studied items may lead to their increase in familiarity-based conjunction errors.
To alter the likelihood that recollection would occur, they manipulated study repetition such that some compound words occurred once whereas others occurred three times (with increased repetitions enhancing the likelihood of recollection). In addition, in Experiment 2, they used a modality manipulation (i.e., words seen or heard) to provide participants with an additional source of information that they could recollect to avoid conjunction errors. Age-related differences were observed for hit rates but not false alarms rates. All participants showed the same pattern of "old" responses across item types (old $>$ conjunction lure $>$ feature lure $>$ new). Age-related differences emerged, however, when retrieval of modality information provided a way to avoid conjunction errors. The results suggest that older adults' inability to recollect study stimuli (i.e., a retrieval-based impairment) lead to increased memory conjunction errors.

Although findings from these behavioral studies show clear age differences in memory conjunction errors, they are ambiguous as to whether age-related memory distortions reflect failures to appropriately bind stimulus elements at study or retrieve the links/associations between stimulus components at test (i.e., representational account) or result from errors regarding the ease in processing of studied elements or familiarity in the absence of recollection (i.e., processing account). Importantly, although the existing literature predominantly casts the representational and processing accounts as mutually exclusive, it is possible that there are circumstances under which individuals may rely more on representations (e.g., when an item is encoded strongly) and other circumstances under which familiarity is important (e.g., weak memory traces). Moreover, even within the same subject, both of these processes could be operating, depending on the strength of the memory representation. As such, agerelated memory conjunction errors may arise from some combination of faulty representational and processing mechanisms.

Finally, regarding brain circuitry, the neural mechanisms underlying age-related memory conjunction errors have been inferred based mainly upon indirect findings from neuropsychological data. Elucidating the neural bases of such errors may provide valuable insight into the core mnemonic processes. For example, two recent neuroimaging studies have explored age-related brain changes during true and false memory formation (Dennis, Kim, \& Cabeza, 2007) and retrieval (Dennis, Kim, \& Cabeza, 2008) using a modified version of the Deese-RoedigerMcDermott paradigm. In this paradigm, participants study lists of words that are semantically related to a word that is not presented (related lure) and, at test, show a strong tendency to incorrectly recognize that related lure (Roediger \& McDermott, 1995). Both neuroimaging studies revealed age-related increases in left middle temporal gyrus for processing of false memories as well as age-related reductions in the medial-temporal lobe (hippocampus) for processing of true memories. This latter finding is consistent with 
a prior study linking reductions in hippocampal activity with older adults' recollection deficits (Daselaar, Fleck, \& Cabeza, 2006; Daselaar, Fleck, Dobbins, Madden, \& Cabeza, 2006). Importantly, these results indicate that the nature of the memory distortion (e.g., gist-based false recognition, memory conjunction error) as well as the paradigm used to elicit the memory errors may determine the locus of agerelated differences in neural activity during false memories.

In the current study, we examined directly the impact of age on the neural basis of memory conjunction error retrieval. To do so, we conducted an event-related fMRI study in young and healthy old adults during retrieval of intact (i.e., direction repetitions), conjunction, feature, and new compound words. To equate overall recognition performance between the young adults and the older adults, we inserted a 2-hr delay between the study and the test sessions for young participants.

In light of the aforementioned evidence and theoretical views of memory conjunction errors, we predicted that compared with young adults, older adults would show reductions in hippocampal activity during retrieval of true memories (i.e., hits > conjunction errors). In addition, we predicted that older adults would exhibit increased recruitment of other brain regions, such as the frontal lobes, during retrieval of true memories, consistent with prior reports demonstrating a shift from hippocampal- to frontal-based processing in healthy older adults during encoding (e.g., Gutchess et al., 2005) and retrieval (e.g., Grady, McIntosh, \& Craik, 2005) of veridical memories. Finally, we investigated the possibility that regions within the parahippocampal gyrus, a medial-temporal lobe region, can be associated with processing of stimulus familiarity (e.g., Daselaar, Fleck, \& Cabeza, 2006) and may play a role in age-related conjunction errors (conjunction false alarms and feature false alarms $>$ hits).

\section{METHODS}

\section{Participant Demographics}

Fifteen young adults between the ages of 19 and 28 years ( $M=22.3$ years, $S D=2.9$ years; 10 women $)$ and 15 older adults between the ages of 66 and 80 years $(M=$ 72.3 years, $S D=4.7$ years; 9 women) were paid for their participation. ${ }^{1}$ Young adults were recruited from flyers posted on the Harvard University campus and older adults were recruited from Cambridge, Massachusetts, and the surrounding communities. All participants were screened to ensure that they were healthy, right-handed, had no contra-indications for fMRI, and were not taking psychotropic medication. Informed consent was obtained from all participants (Figure 1).

\section{Neuropsychological Assessment}

In addition, older adult participants were given a battery of neuropsychological tests to assess their mental functioning

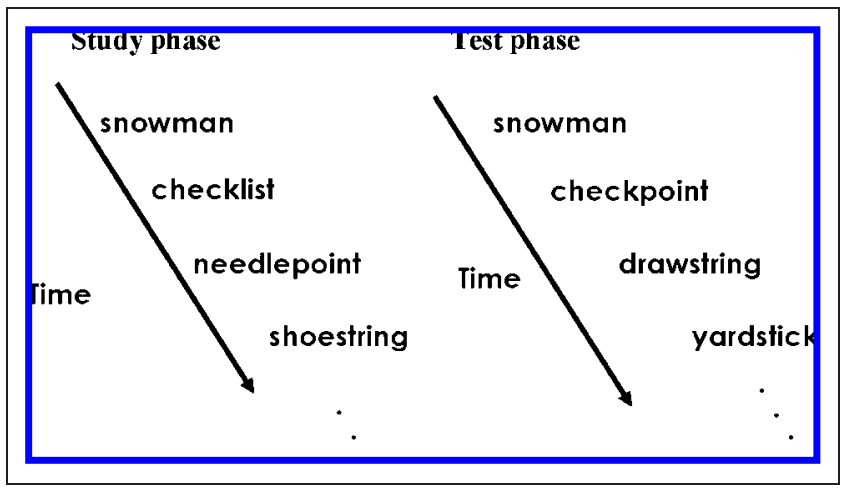

Figure 1. Depiction of experimental protocol (for details, see Methods). At study, participants were shown compound words and instructed to judge whether the word was "pleasant" or "unpleasant." At test, four types of compound words were presented: (i) "intact" words-direct repetitions from the study list; (ii) "conjunction lures"recombinations of studied stimuli; (iii) "feature lures"—one component taken from a studied word and one component novel; and (iv) "new" words-completely novel stimuli. Participants were asked to decide if each compound word was "old" (i.e., appeared during the study phase) or "new" (i.e., novel). Accurate retrieval was defined as an "old" response to an "intact word" (hit), whereas false retrieval was defined as an "old" response to either a "conjunction lure" (conjunction false alarm) or a "feature lure" (feature false alarm).

and to ensure that they were not demented. The neuropsychological battery consisted of the Mini-Mental State Exam, subtests from the Wechsler Adult Intelligence Scale-III (Mental Arithmetic, Visual Paired Associates, Verbal Paired Associates), and subtests from the Wechsler Adult Memory Tests-III (Logical Memories, Mental Control, Digit Span Backward), the California Learning Test, the modified Wisconsin Card Sorting Test, and the Controlled Oral Word Association Test. Participants whose performance was greater than $1 S D$ below the mean were excluded from participation.

\section{Stimuli and Experimental Cognitive Task}

Stimuli were 315 compound words and consisted of 105 triplet sets. Each triplet consisted of two compound words (e.g., courtship, birdhouse) and a third compound word that was a recombination of the other two stimuli (e.g., courthouse).

All participants began with a practice session. Stimuli in the practice session were noncompound words. We chose noncompound words for the practice session to instruct subjects to endorse as "old" only those stimuli that were an exact match to a studied word while reserving the valuable compound word triplets for the actual task.

Both the practice session and the study phase were conducted outside of the scanner. At study, participants viewed one compound word at a time on the computer screen and were asked to decide whether they found the compound word to be pleasant or unpleasant. Participants were told that there was no correct answer and that their 
judgment should reflect their opinion. The study listed consisted of 105 compound stimuli: 21 "old" compound stimuli (e.g., snowman), 42 compound stimuli (e.g., checklist, needlepoint) whose components contributed to the 21 "conjunction lures" in the test phase (e.g., checkpoint), and 42 stimuli (e.g., shoestring) whose components contributed to the 42 "features lures" (e.g., drawstring) in the test phase. Participants were instructed to press "1" to indicate that the word was pleasant and to press the number " 2 " to indicate that the word was unpleasant. For older participants, there was approximately a 15 -min delay period between the study and the test phases during which the participants were placed into the scanner. For young participants, there was a 2-hr delay between the study and the test phases. The delay interval, the duration of which was determined based on pilot data, was used to equate recognition accuracy performance between the young and the old groups.

During the scanned test phase, participants viewed one compound word at a time and were asked to decide whether each word appeared previously. The test list consisted of 105 stimuli, 21 "intact" stimuli (e.g., snowman, direct repetitions from the study list), 21 "conjunction lures" (e.g., checkpoint, recombinations of studied stimulus components), 42 "features lures" (e.g., drawstring, one component taken from a studied word and one component novel), and 21 "new" (e.g., yardstick, completely novel) stimuli. Participants were instructed to press "1" to indicate that the word had appeared previously ("old") and to press the number " 2 " to indicate that the word had not appeared previously ("new").

\section{Imaging Session}

The imaging session lasted $30 \mathrm{~min}$ and included two highresolution T1-weighted (MP-RAGE) structural scans and one functional run, $10 \mathrm{~min} 30 \mathrm{sec}$ in length. The fMRI run contained 21 intact words, 21 recombined lure words, 42 feature lures, and 21 new words. Presentation of these words was jittered with presentation of baseline events, during which participants viewed a fixation cross. Compound words occurred for exactly $4 \mathrm{sec}$, whereas baseline trials occurred for 4,8 , or $16 \mathrm{sec}$. The task was presented using MacStim software (CogState Ltd., Melbourne, Australia).

\section{Imaging Acquisition and Analysis}

\section{Acquisition}

Whole-brain gradient-echo, echo-planar images were collected (twenty-six 3.2-mm slices, repetition time $=2$, echo time $=40$ ) using a 1.5 -T Siemens Avanto scanner while the participants performed the test phase of the cognitive task. Slices were oriented along the long axis of the hippocampus with a resolution of $3.125 \times 3.125 \times 3 \mathrm{~mm}$. Highresolution T1-weighted (MP-RAGE) structural images were collected for anatomic visualization. Stimuli were back projected onto a screen and viewed in a mirror mounted above the participant's head. Responses were recorded using an MR-compatible response box. Head motion was restricted using a pillow and foam inserts.

\section{Analysis}

All preprocessing and data analysis were conducted using SPM 99 (Statistical Parametric Mapping; Wellcome Department of Neurology, UK). Slice acquisition timing was corrected by resampling all slices in time relative to the first slice followed by rigid body motion correction. The functional data were then normalized spatially to the standard T1 Montreal Neurological Institute template. Images were resampled into 3-mm cubic voxels and smoothed spatially with an 8-mm FWHM isotropic Gaussian kernel.

For each participant, on a voxel-by-voxel basis, an eventrelated analysis was first conducted in which all instances of a particular event type were modeled through the convolution with a canonical hemodynamic response function. All participants had at least six instances of every modeled event type (for an example of modeling less than 10 instances per event type, see Chua, Schacter, RandGiovannetti, \& Sperling, 2006). Effects for each event type were estimated using a subject-specific, fixed-effects model. These data were then entered into a second order, randomeffects analysis. Analyses contrasted activation as a function of memory performance (comparing accurate retrieval to false retrieval) using the three trial types (intact, conjunction lure, and feature lure). Regions consisting of at least five contiguous voxels that exceeded the threshold of $p<$ .001 were considered reliable.

Using conjunction analyses (using the masking function in SPM99), we then examined that neural regions were (1) commonly activated by young and older participants during accurate retrieval and (2) differentially activated by young or older participants during accurate and false retrieval. For conjunction analyses examining commonalities between groups, the threshold for each contrast entered in to a conjunction analysis was set at $p<$ .01 , such that the conjoint probability of the conjunction analysis using Fisher's estimate (Lazar, Luna, Sweeney, \& Eddy, 2002; Fisher, 1950) was $p<.001$. For conjunction analyses examining differences between groups, the threshold for the first contrast entered in to a conjunction analysis was set at $p<.01$, whereas the threshold for the second contrast entered into the conjunction analysis was set at $p<.001$ (such that the conjoint probability of the conjunction analysis using Fisher's estimate was $p<.001)$.

All activations are presented in neurological coordinates (i.e., activity in the right hemisphere is presented on the right side of the brain image). Voxel coordinates are reported in Montreal Neurological Institute coordinates and reflect the most significant voxel within the cluster. For young adults, activity is projected onto a composite image 
created by averaging the structural images of all young adult participants. For old adults, activity is projected onto a composite image created by averaging the structural images of all old adult participants.

\section{RESULTS}

\section{Behavioral Data}

The proportion of studied and unstudied stimuli endorsed as "old" are shown in Table 1. An ANOVA with Response Type (hit, conjunction false alarm, feature false alarm, and novel false alarm) as a within-subject factor and Group (young, old) as a between-subject factor revealed a main effect of response type, $F(1,3)=219.20$, $p<.0001$. The main effect of response type stemmed from the fact that intact words were judged "old" at a rate higher than that of conjunction lures, which were identified "old" at a rate higher than that of feature lures, which in turn were identified "old" at a rate higher than that for new words (i.e., old $>$ conjunction $>$ feature $>$ new; all $p$ 's $<.05)$. There was no main effect of group, $F(1,28)<$ 1 , and no Group $\times$ Response Type interaction, $F(1,3)=$ $1.01, p>3$. These findings indicate that the matching procedure successfully equated performance between young and old groups. As such, any age differences observed in the functional imaging analysis cannot be attributable to accuracy differences between the two groups.

An ANOVA conducted on the response latencies associated with making the recognition judgments revealed a main effect of response type, $F(1,3)=5.17, p<.005$. Specifically, response latencies for hits were significantly faster than response latencies to each type of false alarm, all $p$ 's $<.05$. There was no main effect of group, $F(1,28)<$ 1 , and no Group $\times$ Response Type interaction, $F(1,3)<1$.

Table 1.

\begin{tabular}{lcc}
\hline Stimulus Type & Young & Old \\
\hline $\begin{array}{l}\text { A. Proportion of Studied and Unstudied Stimuli Endorsed } \\
\text { as "Old" as a Function of Age }\end{array}$ & $0.78(0.08)$ \\
Intact & $0.82(0.12)$ & $0.41(0.11)$ \\
Conjunction & $0.39(0.14)$ & $0.29(0.16)$ \\
Feature & $0.25(0.12)$ & $0.17(0.10)$ \\
New & $0.18(0.11)$ & \\
& & \\
B. Mean Number of Trials in Each Condition for Each \\
$\quad$ Age Group & $17.3(2.6)$ & $16.4(1.7)$ \\
Intact & $9.2(2.9)$ & $12.0(5.8)$ \\
Conjunction & $10.2(4.1)$ & $3.6(2.1)$ \\
Feature & $3.7(2.4)$ & \\
New & &
\end{tabular}

SDs are shown in parentheses.
Thus, any age differences observed in the functional imaging analysis cannot be attributed to response time differences between the two groups.

\section{Imaging Data}

\section{Neural Regions Associated with Accurate and Distorted Memories in Young Adults}

We assessed regions activated by young adults for accurate (i.e., hits) and distorted (i.e., conjunction lures and features lures) memories. ${ }^{2}$ First, we contrasted activity for hits compared with conjunction lures (see Table 2). This contrast showed bilateral activations in parietal (BA 39/40) and frontopolar regions (BA 10) as well as activity in left middle temporal gyrus (BA 21), right superior temporal gyrus (BA 22), left anterior cingulate (BA 32), right posterior cingulate (BA 31), and right OFC/middle frontal gyrus (BA 9/46). Second, we contrasted activity for hits compared with feature lures (see Table 2). This contrast revealed bilateral activity in superior frontal gyrus (BA 10) and anterior cingulate (BA 24/32) as well as activity in right precuneus (BA 7), right middle temporal gyrus (BA 21), left inferior parietal lobule (BA 40), left posterior cingulate (BA 23), and left parahippocampal gyrus/hippocampus. Finally, we performed a conjunction analysis and contrasted activity for hits compared with conjunction lures and hits compared with feature lures (hit $>$ conjunction lure and hit $>$ feature lure). This analysis revealed bilateral activity in parietal regions (BA 39/40), frontopolar cortex (BA 10), and posterior cingulate (BA 23) as well as activity in right middle temporal gyrus (BA 21) and parahippocampal gyrus/hippocampus. ${ }^{3}$ Of note, no regions were significantly activated for the reverse subtractions/analysis (i.e., conjunction lures $>$ hits or feature lures $>$ hits) at the specified thresholds.

\section{Neural Regions Associated with Accurate and Distorted Memories in Older Adults}

Next, we assessed regions activated by older adults for accurate and distorted memories. First, the contrast of hits compared with conjunction lures revealed activations in right precental gyrus (BA 6) and right OFC (BA 11; Table 3). Second, the contrast of hits compared with feature lures revealed activity in left inferior frontal gyrus (BA 45). Third, the conjunction analysis contrasting activity for hits compared with conjunction lures and hits compared with feature lures (hit $>$ conjunction lure and hit $>$ feature lure) revealed activity in middle temporal gyrus (BA 21). We then assessed activity for the reverse subtractions/analysis to examine neural regions associated with distorted memories. We observed no significant activations for the contrast of conjunctions lures compared with hits. The contrast of activity for feature lures compared with hits revealed activity in right parahippocampal cortex. Likewise, the conjunction analysis for conjunction 
Table 2. Regions of Significant Activation in Young Adults

\begin{tabular}{|c|c|c|c|c|c|}
\hline \multirow[b]{2}{*}{ Region of Activation } & \multirow[b]{2}{*}{ Hemisphere } & \multirow[b]{2}{*}{$B A$} & \multicolumn{3}{|c|}{ MNI Coordinates } \\
\hline & & & $x$ & $y$ & $z$ \\
\hline \multicolumn{6}{|l|}{ Hit $>$ Conjunction FA } \\
\hline \multirow[t]{2}{*}{ Inferior parietal lobule } & $\mathrm{L}$ & 40 & -48 & -42 & 51 \\
\hline & & & -42 & -51 & 57 \\
\hline Parietal lobe, angular gyrus & $\mathrm{R}$ & 39 & 42 & -60 & 24 \\
\hline Middle temporal gyrus & $\mathrm{L}$ & 21 & -54 & -57 & 21 \\
\hline \multirow[t]{3}{*}{ Parietal lobe, angular gyrus } & $\mathrm{L}$ & 39 & -48 & -54 & 27 \\
\hline & & & -51 & -57 & 39 \\
\hline & & & -45 & -60 & 45 \\
\hline Parietal lobe, angular gyrus & $\mathrm{R}$ & 39 & 48 & -51 & 36 \\
\hline Posterior cingulate & $\mathrm{R}$ & 31 & 3 & -42 & 27 \\
\hline Frontopolar, middle frontal gyrus & $\mathrm{L}$ & 10 & -3 & 57 & 0 \\
\hline Frontopolar, superior frontal gyrus & $\mathrm{R}$ & 10 & & 60 & 3 \\
\hline Superior temporal gyrus & $\mathrm{R}$ & 22 & 51 & -9 & 3 \\
\hline Anterior cingulate & $\mathrm{L}$ & 32 & -3 & 42 & -6 \\
\hline Parietal lobe, precuneus & $\mathrm{R}$ & & 3 & -60 & 36 \\
\hline OFC/middle frontal gyrus & $\mathrm{R}$ & $9 / 4$ & 27 & 36 & 33 \\
\hline \multirow[t]{2}{*}{ Insular cortex } & $\mathrm{R}$ & & 45 & -9 & -3 \\
\hline & & & 48 & 0 & -3 \\
\hline \multicolumn{6}{|l|}{ Hit $>$ Feature FA } \\
\hline Frontopolar, superior frontal gyrus & & 10 & -3 & -54 & 6 \\
\hline Parietal lobe, precuneus & $\mathrm{R}$ & 7 & 6 & -54 & 33 \\
\hline Middle temporal gyrus & $\mathrm{R}$ & 21 & 42 & -69 & 21 \\
\hline Parietal lobe, precuneus & $\mathrm{R}$ & 7 & 21 & -54 & 33 \\
\hline Superior temporal & $\mathrm{R}$ & 22 & 48 & -6 & -9 \\
\hline Parahippocampal gyrus/hippocampu & $\mathrm{L}$ & $35 / \mathrm{na}$ & -18 & -18 & -21 \\
\hline Anterior cingulate & $\mathrm{R}$ & 24 & 12 & -18 & 45 \\
\hline Anterior cingulate & $\mathrm{L}$ & 32 & -3 & 45 & -3 \\
\hline Frontopolar, superior frontal gyrus & $\mathrm{R}$ & 10 & 15 & 63 & 6 \\
\hline Inferior parietal lobule & $\mathrm{R}$ & 40 & 51 & -45 & 45 \\
\hline Middle occipital gyrus & $\mathrm{L}$ & 19 & -39 & -75 & 30 \\
\hline Posterior cingulate & $\mathrm{L}$ & 23 & -3 & -39 & 24 \\
\hline \multicolumn{6}{|c|}{ Hit $>$ Conjunction FA and Hit $>$ Feature FA } \\
\hline \multirow[t]{3}{*}{ Parietal, angular gyrus } & $\mathrm{R}$ & 39 & 42 & -60 & 24 \\
\hline & & & 39 & -66 & 30 \\
\hline & & & 48 & -69 & 33 \\
\hline
\end{tabular}


Table 2. (continued)

\begin{tabular}{|c|c|c|c|c|c|}
\hline \multirow[b]{2}{*}{ Region of Activation } & \multirow[b]{2}{*}{ Hemisphere } & \multirow[b]{2}{*}{$B A$} & \multicolumn{3}{|c|}{ MNI Coordinates } \\
\hline & & & $x$ & $y$ & $z$ \\
\hline \multirow[t]{2}{*}{ Inferior parietal lobule } & $\mathrm{R}$ & 40 & 48 & -54 & 39 \\
\hline & & & 54 & -57 & 45 \\
\hline \multirow[t]{2}{*}{ Posterior cingulate } & $\mathrm{R}$ & 23 & 3 & -42 & 27 \\
\hline & & & 3 & -60 & 36 \\
\hline Posterior cingulate & $\mathrm{L}$ & 23 & -6 & -48 & 33 \\
\hline \multirow[t]{3}{*}{ Parietal, angular gyrus } & $\mathrm{L}$ & 39 & -48 & -54 & 27 \\
\hline & & & -39 & -75 & 30 \\
\hline & & & -45 & -69 & 30 \\
\hline \multirow[t]{2}{*}{ Frontopolar, middle frontal gyrus } & $\mathrm{L}$ & 10 & -3 & 57 & 0 \\
\hline & & & -3 & 42 & -6 \\
\hline Frontopolar, superior frontal gyrus & $\mathrm{R}$ & 10 & 6 & 60 & 3 \\
\hline Insular cortex & $\mathrm{R}$ & 13 & 45 & -9 & -3 \\
\hline \multirow[t]{2}{*}{ Inferior parietal lobule } & $\mathrm{L}$ & 40 & -48 & -48 & 45 \\
\hline & & & -48 & -57 & 42 \\
\hline Anterior cingulate & $\mathrm{R}$ & 24 & 6 & -15 & 39 \\
\hline Inferior parietal lobule & $\mathrm{R}$ & 40 & 39 & -45 & 51 \\
\hline Middle temporal gyrus & $\mathrm{R}$ & & 60 & -54 & 18 \\
\hline Parahippocampal gyrus/hippocampus & $\mathrm{R}$ & & 21 & -3 & -21 \\
\hline
\end{tabular}

Regions significant at uncorrected $p<.001$ with an extent $>5$.

$\mathrm{BA}=$ Brodmann's area (approximate) $; \mathrm{MNI}=$ Montreal Neurological Institute.

lures compared with hits and feature lures compared with hits (conjunction lure $>$ hit and feature lure $>$ hit) revealed activity in parahippocampal gyrus.

\section{Neural Regions Commonly Associated with Young and Old during Accurate Retrieval}

We examined shared regions of activation across young and old participants during retrieval of accurate as compared with distorted memories (see Table 4). To do so, we first conducted a conjunction analysis to identify regions that were more active during the "intact" (hits) than during the "conjunction lure" (false alarms) for both young and old groups. This analysis revealed activity in middle temporal gyrus (BA 21) and precental gyrus (BA 4). Next, we conducted the same type of conjunction analysis with hits and feature lures (false alarms). This analysis showed activity in right precuneus, right middle temporal gyrus, and left middle frontal gyrus (BA 10). Finally, we conducted a conjunction analysis to identify regions that were more active during the "intact" condition than during the "conjunction lure" and "feature lure" condition for both young and old groups (hit $>$ conjunction lure and hit $>$ feature lure). This analysis showed activity in right precuneus and right middle temporal gyrus.

\section{Neural Regions Commonly Associated with Young and Old during Inaccurate Retrieval}

No regions were commonly associated with young and old during retrieval of distorted memories as compared with true memories. This result was anticipated because no regions were found to be activated in any of the distorted memory compared with accurate memory contrasts in young adults.

\section{Neural Regions Associated with Retrieval of Accurate Memories as a Function of Age}

We assessed regions uniquely activated by young (i.e., young $>$ old) or older (i.e., old $>$ young) adults for retrieval of accurate as compared with distorted memories (threshold set at $p<.001$, see Analysis section). To do so, we contrasted activity during "intact" (i.e., accurate retrieval) versus "conjunction lure" (i.e., false retrieval) conditions as well as activity during "intact" (i.e., accurate retrieval) versus "feature lure" (i.e., false retrieval) conditions. 
Table 3. Regions of Significant Activation in Older Adults

\begin{tabular}{|c|c|c|c|c|c|}
\hline \multirow[b]{2}{*}{ Region of Activation } & \multirow[b]{2}{*}{ Hemisphere } & \multirow[b]{2}{*}{$B A$} & \multicolumn{3}{|c|}{ MNI Coordinates } \\
\hline & & & $x$ & $y$ & $z$ \\
\hline \multicolumn{6}{|l|}{ Hit $>$ Conjunction FA } \\
\hline Precentral gyrus & $\mathrm{R}$ & 6 & 39 & 0 & 48 \\
\hline OFC & $\mathrm{R}$ & 11 & 18 & 42 & -15 \\
\hline \multicolumn{6}{|l|}{ Hit $>$ Feature FA } \\
\hline Inferior frontal gyrus & $\mathrm{L}$ & 45 & -42 & 36 & 6 \\
\hline \multicolumn{6}{|c|}{ Hit $>$ Conjunction FA and Hit $>$ Feature FA } \\
\hline Middle temporal gyrus & $\mathrm{R}$ & 21 & 45 & -57 & 15 \\
\hline \multicolumn{6}{|l|}{ Conjunction FA $>$ Hit } \\
\hline & & \multicolumn{4}{|c|}{ No significant activations } \\
\hline \multicolumn{6}{|l|}{ Feature FA $>$ Hit } \\
\hline Limbic lobe, isthmus & $\mathrm{R}$ & 27 & 16 & -27 & -12 \\
\hline \multicolumn{6}{|c|}{ Conjunction FA $>$ Hit and Feature FA $>$ Hit } \\
\hline Limbic lobe, isthmus & $\mathrm{R}$ & 27 & 16 & -27 & -12 \\
\hline Parahippocampal gyrus & $\mathrm{R}$ & 35 & 33 & -21 & -24 \\
\hline
\end{tabular}

Regions significant at uncorrected $p<.001$ with an extent $>5$.

$\mathrm{BA}=$ Brodmann's area (approximate); MNI = Montreal Neurological Institute.

The comparison of activity during "intact" versus "conjunction lure" conditions showed that young adults (vs. older adults) activated right amygdala/hippocampus and left caudate during accurate retrieval compared with distorted retrieval (see Figure 2A). Previous studies in young adults have documented hippocampal and/or thalamic activity during the successful recollection of studied stimuli (e.g., Eldridge, Knowlton, Furmanksi, Bookheimer, \& Engel, 2000; Henson, Rugg, Shallice, Josephs, \& Dolan, 1999). The comparison of activity during "intact" versus "feature lure" conditions demonstrated that young adults (vs. older adults) activated right hippocampus and right thalamus during accurate retrieval (see Figure 2B). Of note, the peak of the activity for the "intact" versus "feature lure" contrast was located at a more posterior location on the long axis of the hippocampus than was the peak for the "intact" versus "conjunction lure" comparison. Taken together, these results demonstrate that young adults as compared with older adults activated right anterior hippocampus and amygdala as well as bilateral thalamus during veridical retrieval compared with distorted retrieval.

By contrast, the comparison of activity during "intact" versus "conjunction lure" conditions for older adults (vs. young adults) showed that the elderly activated right infe- rior frontal, right posterior cingulate and left insula during accurate retrieval (see Figure 3A). Similarly, the comparison of activity during "intact" versus "feature lure" conditions demonstrated that older adults (vs. young adults) activated right middle frontal during accurate retrieval (see Figure 3B). Thus, older adults as compared with young adults activated right inferior and middle frontal regions during veridical retrieval as compared with distorted retrieval.

\section{Neural Regions Associated with Retrieval of Distorted Memories as a Function of Age}

To examine the regions that were uniquely activated by young (i.e., young $>$ old) or older (i.e., old $>$ young) adults during retrieval of distorted memories as compared with true memories, we looked for regions in which activity was greater during "conjunction lure" and "feature lure" conditions than during the "intact" condition. For young adults, we observed no regions in which activity was greater during "conjunction lure" and "feature lure" conditions than during the "intact" condition. For older adults, however, right parahippocampal gyrus showed significant activity during "conjunction lure" and "feature lure" conditions than during the "intact" condition (see Figure 4). It should be noted that such activity in right parahippocampal gyrus may have been driven primarily by the feature lure condition, as this condition also elicited

Table 4. Regions of Significant Activation Common to Young and Older Adults

\begin{tabular}{lcrrrrr}
\hline & & & \multicolumn{3}{c}{ MNI } \\
& & & \multicolumn{3}{c}{ Coordinates } \\
\cline { 4 - 7 } Region of Activation & Hemisphere & $B A$ & $x$ & $y$ & $z$ \\
\hline Hit $>$ Conjunction FA & & & & & \\
Middle temporal gyrus & $\mathrm{R}$ & 21 & 42 & -57 & 18 \\
Precentral gyrus & $\mathrm{R}$ & 4 & 42 & -15 & 60 \\
& & & & & \\
Hit $>$ Feature FA & & & & & \\
Parietal lobe, precuneus & $\mathrm{R}$ & 7 & 6 & -51 & 33 \\
& & & 9 & -60 & 33 \\
& & & & & & \\
& & & & 15 & -45 & 30 \\
Middle temporal gyrus & $\mathrm{R}$ & 21 & 45 & -60 & 15 \\
Middle frontal gyrus & $\mathrm{L}$ & 10 & -3 & 45 & -3
\end{tabular}

Hit $>$ Conjunction FA and Hit $>$ Feature FA

\begin{tabular}{llrrrr} 
Parietal lobe, precuneus & $\mathrm{R}$ & 7 & 9 & -57 & 33 \\
Middle temporal gyrus & $\mathrm{R}$ & 21 & 45 & -57 & 15 \\
\hline
\end{tabular}

Regions significant at uncorrected $p<.001$ with an extent $>5$.

$\mathrm{BA}=$ Brodmann's area (approximate) MNI $=$ Montreal Neurological Institute. 
Figure 2. Regions uniquely activated by young adults during retrieval of accurate ("hits") as compared with distorted (A, "conjunction lures"; B, "feature lures") memories. Coordinates are given in standard Montreal Neurological Institute (MNI) space. Activity is projected onto a composite structural image created by averaging the structural images of all young adult participants.
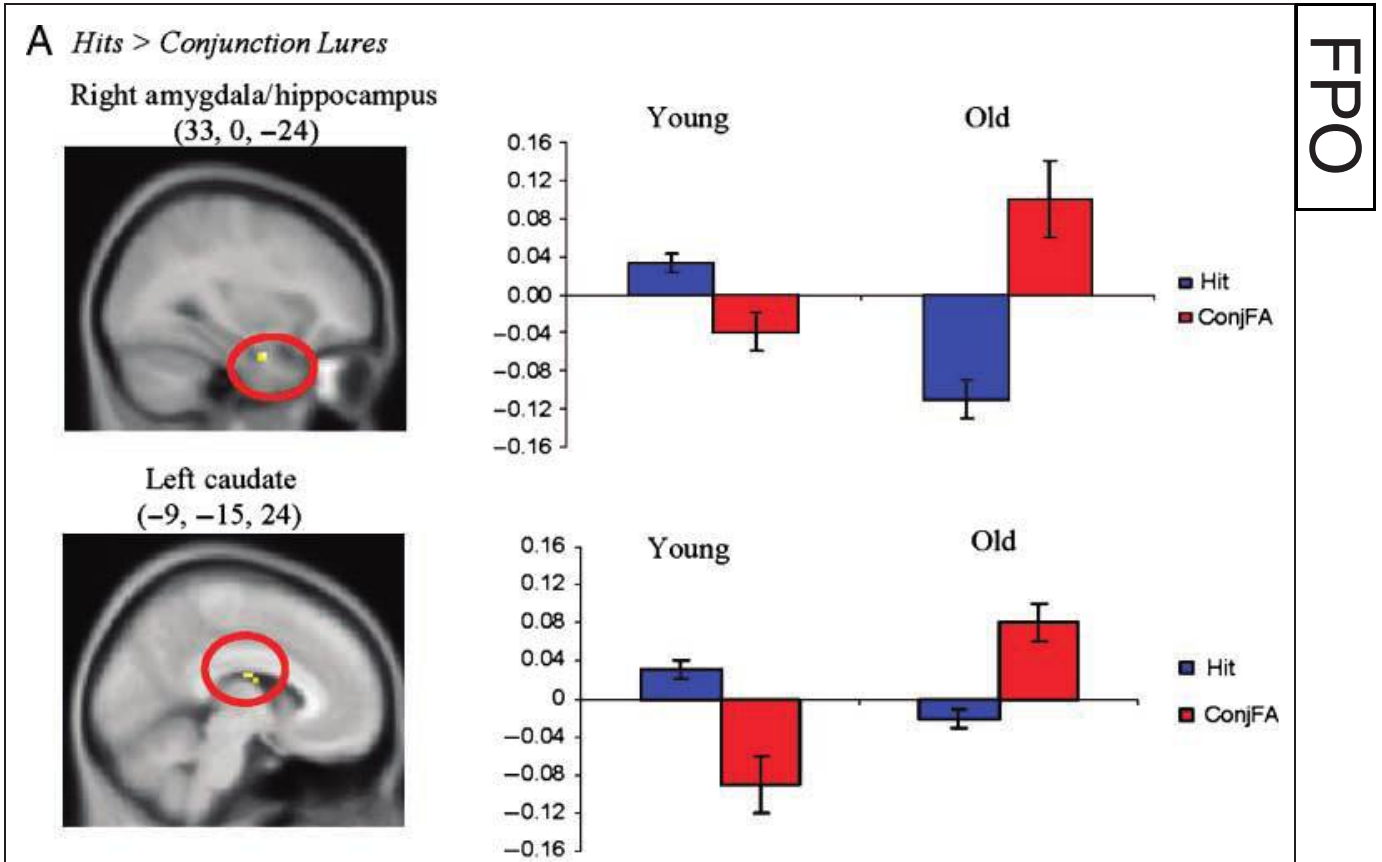

B Hits $>$ Feature Lures
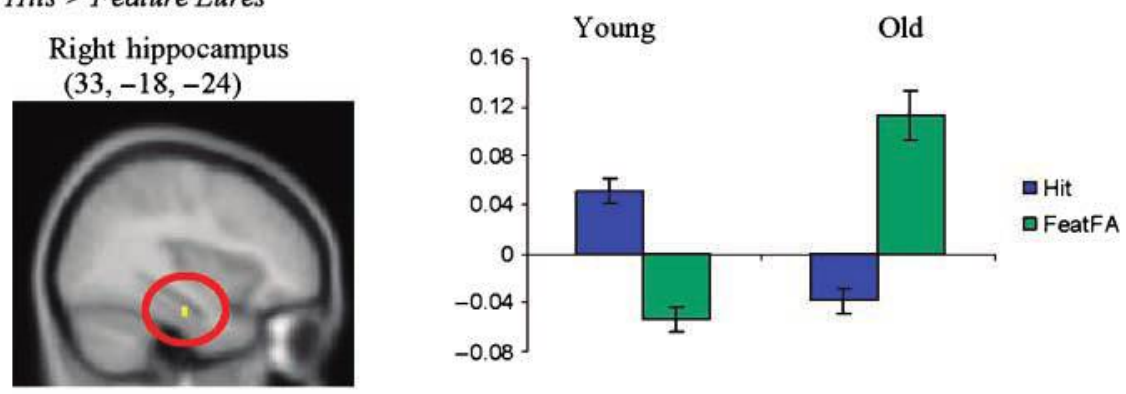

Right thalamus $(6,-12,12)$

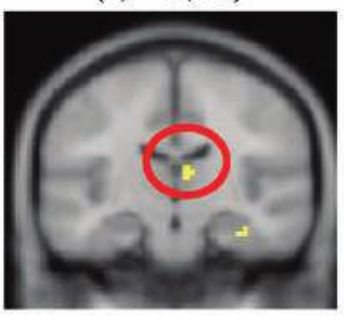

parahippocampal activity in the comparison of distorted versus accurate retrieval (i.e., feature false alarm vs. hits) in older adults.

\section{DISCUSSION}

The current study used the memory conjunction error paradigm and event-related fMRI to examine the cognitive and the neural basis of age-related memory distortions. There were three main findings. First, young adults showed significantly greater activity than older adults in right hippocampus during retrieval of accurate memories. Second, older adults demonstrated greater activity than young adults in right inferior and middle $\mathrm{pFC}$ during accurate retrieval. Finally, the right parahippocampal gyrus showed a stronger relation to false retrieval in older adults than in young adults. We observed no regions in which activity showed a stronger relation to false retrieval in young adults. Below we consider each of these findings.

\section{Age-related Neural Differences during Veridical Retrieval}

In regard to accurate retrieval, young adults showed significantly greater activity than older adults in right hippocampus (Figure 2). Activity in the hippocampus has been 
Figure 3. Regions uniquely activated by old adults during retrieval of accurate ("hits") as compared with distorted (A, "conjunction lures"; B, "feature lures") memories. Coordinates are given in standard Montreal Neurological Institute (MNI) space. Activity is projected onto a composite structural image created by averaging the structural images of all older adult participants.

A Hits $>$ Conjunction Lures

Inferior frontal gyrus

$(45,24,12)$

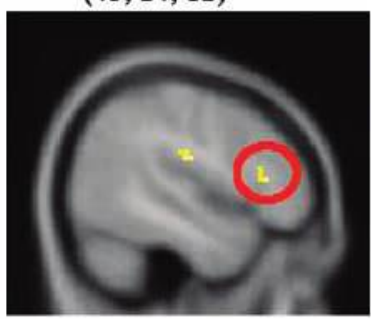

Posterior cingulate $(18,-54,9)$

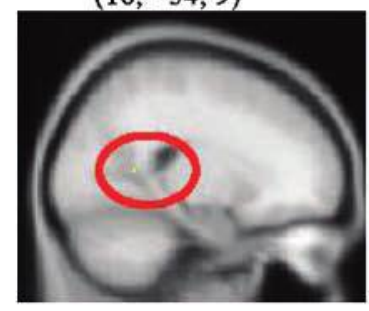

Insula

$(48,-18,21)$

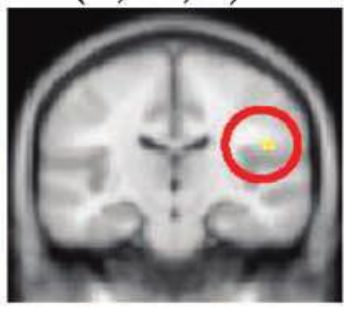

B Hits $>$ Features Lures

Middle frontal gyrus

$(45,42,-6)$

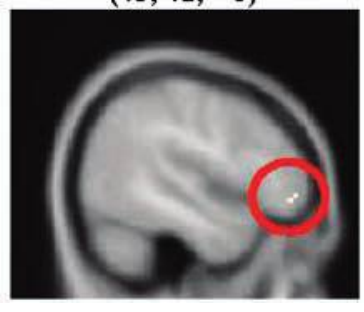

Old
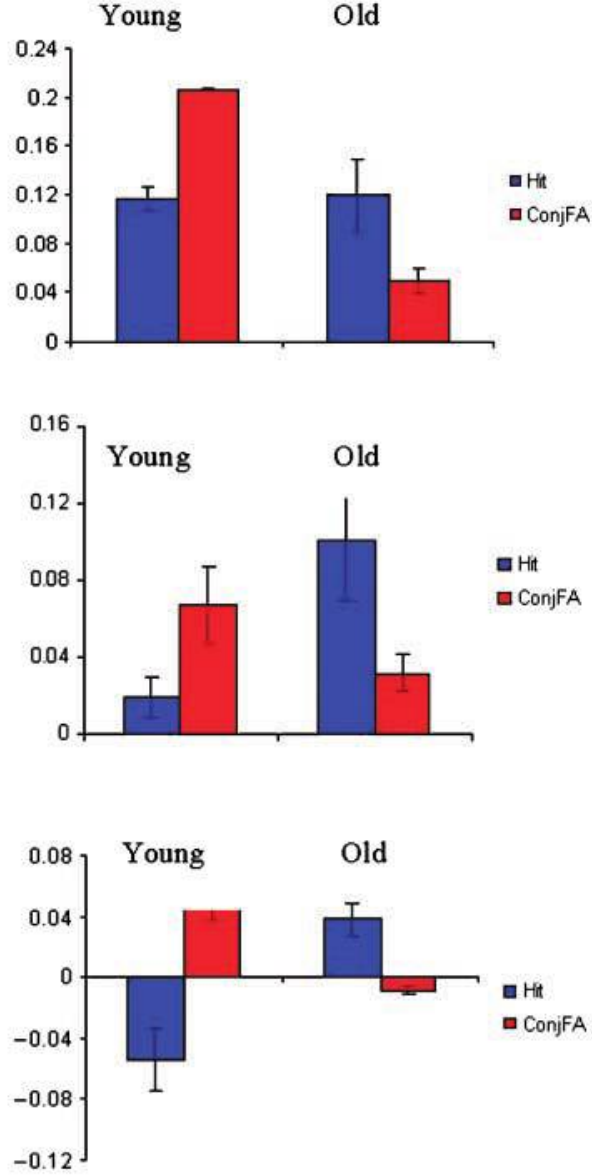

ConjFA

a Hit - Fe atFA
Figure 4. Regions uniquely activated by old adults during retrieval of distorted as compared with accurate memories. Coordinate is given in standard Montreal Neurological Institute (MNI) space. Activity is projected onto a composite structural image created by averaging the structural images of all older adult participants.
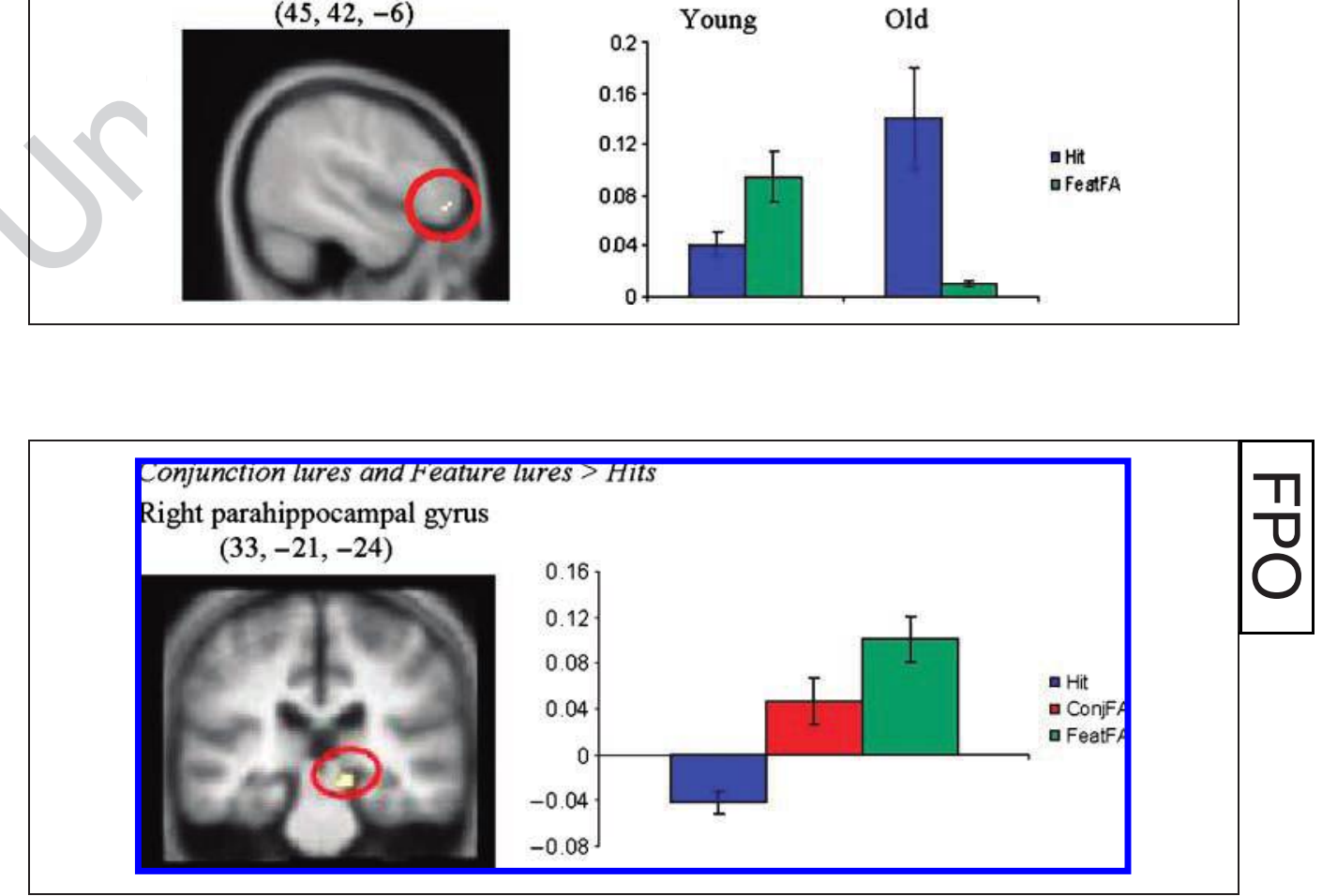
linked to the conscious recollection of learned episodes (e.g., Eldridge et al., 2000; Schacter, Alpert, Savage, Rauch, \& Albert, 1996) or to retrieval of associations between components of an event (e.g., Giovanello, Schnyer, \& Verfaellie, 2004, 2008; Preston, Shrager, Dudukovic, \& Gabrieli, 2002). As such, the age-related decreases in hippocampal activity observed in the current study may reflect declines in recollection processes or decrements in associative memory. Indeed, age-related reductions in recollection (Bastin \& Van der Linden, 2003; Davidson \& Glisky, 2002; Parkin \& Walter, 1992) and associative memory (for a review, see Old \& Naveh-Benjamin, 2008) have been reported by several investigators, and more recently, older adults' recollection deficits have been linked to reductions in hippocampal activity (Daselaar, Fleck, \& Cabeza, 2006; Daselaar, Fleck, Dobbins, et al., 2006).

Interestingly, the hippocampal activity observed in the current study was located in an anterior region of the hippocampus. Such anterior hippocampal activity has been observed during successful encoding (Chua, Schacter, Rand-Giovennetti, \& Sperling, 2007) and retrieval (Giovanello et al., 2004, 2008) of associations as well as when participants imagine future events by recombining elements of past experiences (Addis \& Schacter, 2008). Behaviorally, older adults show deficits in memory specificity when retrieving past events and imagining future events (Addis, Wong, \& Schacter, 2008). Taken together, these findings suggest a relationship between the anterior hippocampal activity and the operation of adaptive, constructive mnemonic processes (Schacter \& Addis, 2009).

In contrast to the young adult findings, older adults demonstrated greater activity than young adults during accurate retrieval in right inferior and middle pFC. Such a shift from hippocampal- to frontal-based processing in healthy older adults has been documented previously during episodic encoding (e.g., Gutchess et al., 2005) and veridical episodic retrieval (e.g., Grady et al., 2005). In these studies, significant correlations were obtained between decreased hippocampal activity and increased prefrontal activity during accurate performance. These results have been interpreted to reflect compensatory processes whereby prefrontal regions are recruited to counteract neurocognitive decline in the context of deficient hippocampal system function.

Indeed, several laboratories have reported that recruitment of prefrontal regions is associated with successful memory in older adults (e.g., Grady et al., 2005; Morcom, Good, Frackowiak, \& Rugg, 2003; Cabeza, Anderson, Locantore, \& McIntosh, 2002; Rosen et al., 2002), leading to the suggestion that age-related increased recruitment of unique brain regions may reflect a compensatory process. Of note, the increased prefrontal activity (in the context of decreased hippocampal activity) reported by Grady et al. (2005) occurred under conditions in which performance was not matched between young and older adults. In the current study, however, we used a matching procedure to equate performance between the young and the older groups yet observed increased activity in right inferior and middle pFC in an analysis contrasting hits and errors. This finding suggests that group differences in behavioral performance are not a prerequisite for observing age-related recruitment of prefrontal regions during accurate retrieval.

Finally, two regions were commonly associated with young and old during the retrieval of accurate memories: right precuneus and right superior temporal gyrus. Activity in both right precuneus and right superior temporal gyrus has been observed in previous studies of veridical retrieval (Cabeza et al., 1997; Fletcher, Shallice, Frith, Frackowiak, \& Dolan, 1996). In the current study, engagement of right superior temporal gyrus, an important region for the processing of speech, may reflect participants' covert generation of the sounds of studied stimuli, whereas activity in right precuneus may have arisen if participants were mentally picturing the studied word form. In both cases, such strategies may help to guide veridical retrieval of studied items.

\section{Age-related Neural Differences during False Retrieval}

Behaviorally, both young and older adults showed the identical pattern of "old" responses to items (intact $>$ conjunction lure $>$ feature lure $>$ new). This pattern of performance has been observed previously (Jones \& Jacoby, 2005; Rubin et al., 1999) and likely reflects participants' sensitivity to the familiarity of studied stimulus elements. That is, participants are more likely to respond "old" at recognition to conjunction lures that consist of two studied components, to feature lures that consist of one studied component, and to novel items that consist of no studied components.

In the current study, behavioral performance was equated between young and older adults by testing older adults immediately following encoding and testing young adults following a 2-hr delay. Such a matching procedure may have qualitatively changed the memory process for young adults, requiring them to rely somewhat more on familiarity than they otherwise would have done (i.e., had they been tested immediately). It would be of interest for future investigations to examine this issue.

Although there have been extensive discussions about the role of the parahippocampal gyrus in episodic retrieval (for a recent review, see Diana, Yonelinas, \& Ranganath, 2007), our results are consistent with evidence suggesting that parahippocampal activity can be associated with processing episodic familiarity signals (Daselaar, Fleck, \& Cabeza, 2006) and that reliance on familiarity signals within the medial-temporal lobe may be increased with age (Daselaar, Fleck, Dobbins, et al., 2006).

Daselaar, Fleck, and Cabeza (2006) reported a triple dissociation in the medial-temporal lobes in which the anterior hippocampal region and the rhinal cortex exhibited novelty-related activity, the posterior half of the 
hippocampus demonstrated recollection-related activity, and the parahippocampal cortex showed familiarity-related activity. More specifically, activity in posterior parahippocampal gyrus exhibited a continuous increase in activity with increasing levels of stimulus oldness. The finding that posterior parahippocampal activity is linked to stimulus familiarity is consistent with prior fMRI studies demonstrating parahippocampal activity during item-based perceptual retrieval processes (Goh et al., 2004; Cabeza, Rao, Wagner, Mayer, \& Schacter, 2001). In their discussion, Daselaar, Fleck, and Cabeza (2006) and Daselaar, Fleck, Dobbins, et al. (2006) noted that the posterior parahippocampal gyrus receives direct input from several unimodal and polymodal cortical regions (visual, parietal, and temporal cortices) and provides approximately one third of the sensory input into the hippocampus (Suzuki \& Amaral, 1994). This fact, coupled with the idea that familiarity depends more heavily on perceptual processing than on recollection (Yonelinas, 2002), suggests that increases in posterior parahippocampal gyrus with oldness may reflect an increased reliance on perceptual fluency during familiarity-based recognition.

In the current study, we observed increased activity in parahippocampal gyrus in older adults for distorted retrieval than accurate retrieval. Specifically, the comparison of feature lures greater than hits as well as the conjunction analysis (feature and conjunction lures greater than hits) revealed activity in the right parahippocampal gyrus. The engagement of parahippocampal gyrus suggests that older adults may have over relied on the perceptual familiarity of stimulus elements, which led to incorrect endorsement of conjunction and features lures. Such findings indicate that older adults may retrieve information that is less relational (or more familiarity based) than the information young adults retrieve. Nonetheless, older adults can achieve the same level of performance as young adults through successful monitoring of retrieved information via recruitment of prefrontal regions.

It should be noted that even young adults made about as many false alarms as did older adults, we did not find any neural activity for young adults in the false greater than true memory comparisons at our specified significance threshold. At lower thresholds, however, several regions emerged, including bilateral insula and inferior $\mathrm{pFC}$ as well as medial pFC. Future research will be required to determine whether these findings are meaningful or simply the result of chance. Perhaps stronger effects are observed in older adults because there was a dominant processes driving their performance (i.e., reliance on parahippocampal familiarity signals), whereas false memories in younger adults might reflect the influence of a number of processes, including familiarity, misrecollection, and monitoring failures.

The current study focused on one form of memory distortion (i.e., conjunction errors). However, memory distortions can take several forms. Individuals can incorrectly endorse an entirely novel stimulus or a stimulus composed of previously presented components as "old" (i.e., memory conjunction error); incorrectly accept a thematically related yet nonstudied stimulus as "old" (i.e., gist-based false recognition; Koutstaal \& Schacter, 1997); or indicate that an imagined event was perceived or performed (i.e., reality monitoring error; Johnson \& Raye, 1981).

As described in the Introduction, two recent neuroimaging studies have examined age-related brain changes during true and false memory formation (Dennis et al., 2007) and retrieval (Dennis et al., 2008) using a modified version of the Deese-Roediger-McDermott paradigm (i.e., gistbased false recognition). Both neuroimaging studies reported age-related reductions in the medial-temporal lobe (hippocampus) for processing of true memories. This finding is consistent with the age-related differences in hippocampal activity observed in the current study. The prior reports, however, documented age-related increases in left middle temporal gyrus for processing of false memories. This activity was interpreted as an increased reliance on semantic gist by older adults. In the current study, we observed age-related increases in right parahippocampal gyrus during false retrieval, possibly reflecting an over reliance on stimulus familiarity by older adults. Taken together, these results suggest that the nature of the memory distortion (i.e., false recognition, memory conjunction error, gist-based false recognition, or reality monitoring error) as well as the paradigm used to elicit the memory errors may determine the locus of age-related differences in neural activity during false memories. Future studies aimed at directly comparing neural activity elicited for different forms of memory distortions will serve to further clarify the nature of age-related increases in memory errors.

\section{Conclusion}

We used the memory conjunction error paradigm to examine age-related neural differences during retrieval of true and false memories. Young adults compared with older adults recruited the hippocampus during true memory retrieval, consistent with a role for this structure in the retrieval of recollected or associative information. Older adults compared with young adults showed increased recruitment of prefrontal regions during veridical retrieval. Such increased prefrontal activity in older adults has been observed previously and may reflect a compensatory mechanism. Performance in the current study was matched between young and older adults. The findings indicate that a difference in behavioral performance between age groups is not a prerequisite for observing agerelated increase in prefrontal activity during retrieval.

Although we observed no regions in which activity was greater during false than during accurate retrieval for young adults, older adults showed significantly greater activity during false than during accurate retrieval in the right parahippocampal gyrus. This result likely reflects an over reliance by older adults on the familiarity of stimulus elements and points to the importance of the nature of memory distortions as a contributor to the locus of age-related 
differences in neural activity during false memories. Finally, our findings suggest that older adults' memory conjunction errors can be caused by faulty hippocampal retrieval processes.

\section{UNCITED REFERENCES}

Henke, Weber, Kneifel, Wieser, \& Buck, 1999

Koutstaal \& Schacter, 2001

\section{Acknowledgments}

We thank Chris Moore for assistance in identifying stimulus materials and an anonymous reviewer for helpful comments. This work was supported by grants AG023439 from the National Institute on Aging to K. S. G., MH070199 from the National Institute of Mental Health to E. A. K., and AG08441 from the National Institute on Aging to D. L. S.

Reprint requests should be sent to Kelly S. Giovanello, Department of Psychology and Biomedical Research Imaging Center, The University of North Carolina at Chapel Hill, NC, or via e-mail: kgio@unc.edu.

\section{Notes}

1. An additional 7 young adults $(M=24.7$ years, $S D=5.1$ years; 6 women) and 6 older adults $(M=71.0$ years, $S D=3.2$ years; 3 women) participated in the experiment. Data from these individuals could not be analyzed because these participants did not commit the minimum number of false alarms per condition (i.e., $\geq 6$ ).

2. The term "lure" in these analyses is shorthand for false alarms to the particular type of lure indicated.

3. The conjunction analyses were conducted by thresholding each of the two individual contrasts (e.g., hits $>$ conjunctions, hits $>$ feature) at $p<.01$, resulting in a joint probability of $p<$ .001 (using Fisher's estimate, as is standard for this type of conjunction analysis). Because the original contrasts (e.g., hits $>$ conjunctions) were reported at a threshold of $p<.001$, there can be regions (such as the MTL) that appear in the conjunction but not in the individual contrasts. This difference in the regions revealed is due to the thresholding differences; for the hits $>$ conjunctions contrast, the MTL is not revealed at a threshold of $p<.001$ but is at a threshold of $p<.01$.

\section{REFERENCES}

Addis, D. R., \& Schacter, D. L. (2008). Constructive episodic simulation: Temporal distance and detail of past and future events modulate hippocampal activity. Hippocampus, 18, 227-237.

Addis, D. R., Wong, A., \& Schacter, D. L. (2008). Age-related changes in the episodic simulation of future events. Psvchological Science, 18, 33-41.

Cabeza, R., Anderson, N. D., Locantore, J. K., \& McIntosh, A. R. (2002). Aging gracefully: Compensatory brain activity in high-performing older adults. Neuroimage, 17, 1394-1402.

Cabeza, R., Mangels, J., Nyberg, L., Habib, R., Houle, S., McIntosh, A. R., et al. (1997). Brain regions differentially involved in remembering what and when. Neuron, 19, 863-870.

Cabeza, R., Rao, S. M., Wagner, A. D., Mayer, A. R., \& Schacter, D. L. (2001). Can medial temporal lobe regions distinguish true from false? An event-related functional MRI study of veridical and illusory recognition memory. Proceedings of the National Academy of Sciences. U.S.A., 98, 4805-4810.

Chua, E., Schacter, D. L., Rand-Giovennetti, E., \& Sperling, R. A. (2007). Evidence for a specific role of the anterior hippocampal region in successful associative encoding. Hippocampus, 17, 1071-1080.

Chua, E. F., Schacter, D. L., Rand-Giovannetti, E., \& Sperling, R. A. (2006). Understanding metamemory: Neural correlates of the cognitive process and subjective level of confidence in recognition memory. Neuroimage, 29, 1150-1160.

Daselaar, S. M., Fleck, M. S., \& Cabeza, R. (2006). Triple dissociation in the medial temporal lobes: Recollection, familiarity, and novelty. Iournal of Neuropbvsiology 96, 1902-1911.

Daselaar, S. M., Fleck, M. S., Dobbins, I. G., Madden, D. J., \& Cabeza, R. (2006). Effects of healthy aging on hippocampal and rhinal memory functions: An event-related fMRI study. Cerebral Cortex, 16, 1771-1782.

Dennis, N. A., Kim, H., \& Cabeza, R. (2007). Effects of aging on true and false memory formation: An fMRI study. Neuropsvchologia, 45, 3157-3166.

Dennis, N. A., Kim, H., \& Cabeza, R. (2008). Age-related differences in brain activity during true and false memory retrieval. Journal of Cognitive Neuroscience, 20, 1390-1402.

Dodson, C. S., Koutstaal, W., \& Schacter, D. L. (2001). Escape from illusion: Reducing false memories. Trends in Cognitive Science, 4, 391-397.

Dorfman, J. (1994). Sublexical components in implicit memory for novel words. Journal of Experimental Psychology, 20, 1108-1125.

Eldridge, L. L., Knowlton, B. J., Furmanksi, C. S., Bookheimer, S. Y., \& Engel, S. A. (2000). Remembering episodes: A selective role for the hippocampus during retrieval. Nature Neuroscience, 3, 1149-1152.

Fisher, R. A. (1950). Statistical methods for research workers. London: Oliver and Boyd.

Fletcher, P. C., Shallice, T., Frith, C. D., Frackowiak, R. S. J., \& Dolan, R. J. (1996). Brain activity during memory retrieval: The influence of imagery and semantic cuing. Brain. 119, 1587-1596.

Giovanello, K. S., Schnyer, D., \& Verfaellie, M. (2004). A critical role for the anterior hippocampus in relational memory: Evidence from an fMRI study comparing associative and item recognition. Hippocampus, 14, 5-8.

Giovanello, K. S., Schnyer, D., \& Verfaellie, M. (2008). Distinct hippocampal regions make unique contributions to relational memory. Hippocampus.

Goh, J. O., Siong, S. C., Park, D., Gutchess, A., Hebrank, A., \& Chee, M. W. (2004). Cortical areas involved in object, background, and object-background processing revealed with functional magnetic resonance adaptation. Journal of Neuroscience, 24, 10223-10228.

Grady, C., McIntosh, A. R., \& Craik, F. I. M. (2005). Task-related activity in prefrontal cortex and its relation to recognition memory performance in young and old adults. Neuropsvchologia, 43, 1466-1481.

Gutchess, A., Welsh, R. C., Hedden, T., Bangert, A., Minear, M., Liu, L. L., et al. (2005). Aging and the neural correlates of successful picture encoding: Frontal activations compensate for decreased-medial temporal lobe activity. Iournal of Cognitive Neuroscience, 17, 84-96.

Henke, K., Weber, B., Kneifel, S., Wieser, H. G., \& Buck, A. (1999). Human hippocampus associates information in memory. Proceedings of the National Academv of Sciences. U.S.A., 11, 5884-5889. 
Henson, R. N., Rugg, M. D., Shallice, T., Josephs, O., \& Dolan, R. J. (1999). Recollection and familiarity in recognition memory: An event-related functional magnetic resonance imaging study. Journal of Neuroscience, 19, 3962-3972.

Jacoby, L. L., \& Rhodes, M. G. (2006). False remembering in the aged. Current Directions in Psychological Science, 15, 49-53.

Jha, A. P., Kroll, N. E. A., Baynes, K., \& Gazzaniga, M. S. (1997). Memory encoding following complete callosotomy. Journal of Cognitive Neuroscience, 9, 143-159.

Johnson, M. K., \& Raye, C. L. (1981). Reality monitoring. Psychological Review, 88, 67-85.

Jones, T. C., \& Jacoby, L. L. (2005). Conjunction errors in recognition memory: Modality free-errors for older but not for you adults. Acta Psychologica, 120, 55-73.

Jones, T. C., Jacoby, L. L., \& Gellis, L. A. (2001). Cross-modal feature and conjunction errors in recognition memory. Journal of Memory and Language, 44, 131-152.

Koutstaal, W., \& Schacter, D. L. (2001). Memory distortion and aging. In M. Naveh Benjamin, M. Moscovitch, \& H. L. Roediger (Eds.), Perspectives on human memory and cognitive aging: Essays in honour of Fergus Craik. New York: Psychology Press.

Kroll, N. E. A., Knight, R. T., Metcalfe, L., Wolf, E. S., \& Tulving, E. (1996). Cohesion failure as a source of memory illusions. Journal of Memory and Language, 35, 176-196.

Lazar, N. A., Luna, B., Sweeney, J. A., \& Eddy, W. F. (2002). Combining brains: A survey of methods for statistical pooling of information. Neuroimage, 16, 538-550.

Morcom, A. M., Good, C. D., Frackowiak, R. S., \& Rugg, M. D. (2003). Age effects on the neural correlates of successful memory encoding. Brain, 126, 213-229.

Old, S. R., \& Naveh-Benjamin, M. (2008). Differential age effects on item and associative measures of memory: A meta-analysis. Psychology and Aging, 23, 104-118.

Preston, A. R., Shrager, Y., Dudukovic, N. M., \& Gabrieli, J. D. E. (2002). Hippocampal contribution to novel use of relational information in declarative memory. Hippocampus, 14, 148-152.

Reinitz, M. T., Lammers, W. J., \& Cochran, B. P. (1992). Memory-conjunction errors: Miscombinations of stored stimulus features can produce illusions of memory. Memory and Cognition, 20, 1-11.

Reinitz, M. T., Verfaellie, M., \& Milberg, W. P. (1996). Memory conjunction errors in normal and amnesic subjects. Journal of Memory and Language, 35, 286-289.
Roediger, H. L., \& McDermott, K. B. (1995). Creating false memories: Remembering words not present in lists. Journal of Experimental Psychology: Learning, Memory, and Cognition, 21, 803-814.

Roediger, H. L., \& McDermott, K. B. (2000). Tricks of memory. Current Directions in Psychological Science, 9, 123-127.

Rosen, A. C., Prull, M. W., O'Hara, R., Race, E. A., Desmond, J. E., Glover, G. H., et al. (2002). Variable effects of aging on frontal lobe contributions to memory. NeuroReport, 13, 2425-2428.

Rubin, R., Van Patten, C., Glisky, E. L., \& Newberg, W. M. (1999). Memory conjunction errors in young and older adults: Event-related potential and neuropsychological data. Cognitive Neuropsychology, 16, 459-488.

Schacter, D. L. (2001). The seven sins of memory: How the mind forgets and remembers. New York: Houghton Mifflin Company.

Schacter, D. L., \& Addis, D. R. (2009). On the nature of medial temporal lobe contributions to the constructive simulation of future events. Philosophical Transactions of the Royal Society of London, Series B, Biological Sciences, 364, $1245-1253$.

Schacter, D. L., Alpert, N. M., Savage, C. R., Rauch, S. L., \& Albert, M. (1996). Conscious recollection and the human hippocampal formation: Evidence from positron omission tomography. Proceedings of the National Academy of Sciences, U.S.A., 93, 321-325.

Schacter, D. L., \& Slotnick, S. D. (2004). The cognitive neuroscience of memory distortion. Neuron, 44, 149-160.

Suzuki, W. A., \& Amaral, D. G. (1994). Perirhinal and parahippocampal cortices of the macaque monkey: Cortical afferents. Journal of Comparative Neurology, 350, 497-533.

Underwood, B. G. (1965). False recognition produced by implicit verbal responses. Journal of Experimental Psychology, 70, 122-129.

Underwood, B. J., Kapelak, S. M., \& Malmi, R. A. (1976). Discrete verbal units in recognition memory. Journal of Experimental Psychology: Human Learning and Memory, 2, 293-300.

Underwood, B. J., \& Zimmerman, J. (1973). The syllable as a source of error in multisyllable word recognition. Journal of Verbal Learning and Verbal Behavior, 12, 338-344.

Yonelinas, A. P. (2002). The nature of recollection and familiarity: A review of 30 years of research. Journal of Memory and Language, 46, 441-517. 